\title{
Are welders more at risk of respiratory infections? Findings from a cross-sectional survey and analysis of medical records in shipyard workers: the WELSHIP project
}

\author{
Andrea Marongiu, ${ }^{1,2}$ Omar Hasan, ${ }^{3}$ Anila Ali, $^{3}$ Sharoon Bakhsh, ${ }^{3}$ Bobby George, ${ }^{3}$ \\ Nabeel Irfan, ${ }^{3}$ Cosetta Minelli, ${ }^{1}$ Cristina Canova, ${ }^{1,4}$ Susie Schofield, ${ }^{1}$ \\ Sara De Matteis, ${ }^{1,2}$ Paul Cullinan ${ }^{1,2}$
}

\begin{abstract}
${ }^{1}$ Department of Occupational and Environmental Medicine, National Heart \& Lung Institute, Imperial College London, London, UK ${ }^{2}$ MRC-PHE Centre for Environment \& Health, St Mary's Campus, Imperial College London, London, UK

${ }^{3}$ Medical Department, DryDocks World Dubai, Dubai, UAE

${ }^{4}$ Department of Molecular Medicine, University of Padova, Padova, Italy
\end{abstract}

\section{Correspondence to} Paul Cullinan, Imperial College (NHLI) London, Emmanuel Kaye Building G51, 1b Manresa Rd, London SW3 6LR, UK;

p.cullinan@imperial.ac.uk

Received 7 October 2015 Revised 27 January 2016 Accepted 4 February 2016 Published Online First 30 March 2016

\section{SLinked}

http://dx.doi.org/10.1136/ thoraxjnl-2016-208464

\section{CrossMark}

To cite: Marongiu $A$, Hasan 0, Ali A, et al. Thorax 2016;71:601-606.

\section{ABSTRACT}

Background Exposure to welding fume increases the risk of pneumococcal infection; whether such susceptibility extends to other respiratory infections is unclear. We report findings from a survey and from medical consultation data for workers in a large shipyard in the Middle East.

Methods Between January 2013 and December 2013, we collected cross-sectional information from 529 male workers variously exposed to welding fume. Adjusted ORs for respiratory symptoms (cough, phlegm, wheezing, shortness of breath and 'chest illness') were estimated using multivariable logistic regression. Subsequently, we examined consultation records from 2000 to 2011 for 15954 workers who had 103840 consultations for respiratory infections; the associations between respiratory infections and levels of welding exposure were estimated using a count regression model with a negative binomial distribution.

Results $13 \%$ of surveyed workers reported respiratory symptoms with a higher prevalence in winter, particularly among welders. The adjusted OR in welders versus other manual labourers was $1.72(95 \% \mathrm{Cl} 1.02$ to 3.01 ) overall and 2.31 (1.05 to 5.10) in winter months; no effect was observed in summer. The risk of consultation for respiratory infections was higher in welders than in manual labourers, with an adjusted incidence rate ratio of 1.45 (1.59 to 1.83$)$ overall, 1.47 (1.42 to 1.52) in winter and 1.33 (1.23 to 1.44 ) in summer (interaction, $p<0.001$ ).

Conclusions The observation that respiratory symptoms and consultations for respiratory infection in welders are more common in winter may indicate an enhanced vulnerability to a broad range of infections. If confirmed, this would have important implications for the occupational healthcare of a very large, global workforce.

\section{INTRODUCTION}

Successive analyses of occupational mortality in England and Wales have indicated an increased risk of death from pneumococcal pneumonia in men whose recorded occupation was in welding; that the risk may be reversible is suggested by the observation that it is present only in men of working

\section{Key messages}

What is the key question?

- Does the susceptibility of welders to pneumococcal disease extend to other, non-pneumococcal respiratory infections?

\section{What is the bottom line?}

- At both cross-sectional survey and through an examination of medical consultations, shipyard welders appear to have an increased risk of seasonal, respiratory infections.

\section{Why read on?}

- A heightened risk of a broad range of respiratory infections in welders would have important implications for their occupational healthcare.

age. ${ }^{1}$ Supportive, if weaker, evidence derives from an analysis of patients with invasive pneumococcal disease in Canada ${ }^{2}$ and a small case series of deaths from pneumonia in welders in Norway. ${ }^{3}$ Among construction workers in Sweden there was an increased risk of death from 'infectious pneumonia' (lobar and other) in those with exposure to metal fume. ${ }^{4}$ In 2003, Palmer and colleagues ${ }^{5}$ published a case-control analysis of the occupational exposures of men admitted to a hospital in England with pneumonia. An association with recent occupational exposure to metal fume was evident for men with either lobar or subsegmental pneumonia; the associations were strongest for exposure to ferrous fume and were reported for those with pneumococcal disease and those with infections attributed to Mycoplasma, Legionella or Haemophilus species.

There is thus robust evidence that welders and others with occupational exposure to metal fume have a heightened, probably reversible, susceptibility to pneumococcal disease. The evidence that this extends to respiratory infections caused by other organisms is more tenuous. The WELSHIP project was set up to investigate the effects of arc-welding 
fume on cardiorespiratory health in shipyard welders; here we present those findings that pertain to the issue of respiratory infections.

\section{METHODS}

The shipyard is located in the Middle East and is one of the world's largest repair and refit facilities of oil and gas tankers. It employs at any one time around 7000 men and women from around 50 countries, principally India, Pakistan, Bangladesh and the Philippines; they include approximately 4000 men whose work is solely or partly arc welding, usually on mild steel base metal. The information analysed here was collected from two of the studies in WELSHIP; a cross-sectional survey and a retrospective cohort study of medical consultation records.

\section{Cross-sectional survey}

In late 2012, from the yard's payroll, we identified all current employees who had been employed for at least 1 year $(n=6217)$ and excluded all those with office jobs $(n=549)$ or work that involved probable exposure to fine dusts, solvents or smoke $(n=964)$, those whose country of origin was other than those listed above $(n=250)$ and those whose expected end date of employment was before $2013(n=59)$. From the remaining 4276 employees we selected for the cross-sectional survey a sample of 566 men who were frequency matched for age and country of origin across four levels of probable exposure to welding fume (welders, platers, pipe-fitters and non-welding shipyard manual workers) based on their job titles; of these, $529(93 \%)$ agreed to take part.

The survey included a lung function test, arterial stiffness measurements and a brief questionnaire that enquired, in one of five languages (Bangla, Malayalam, Tamil, Tagalog or Urdu), into a range of current respiratory symptoms (cough, phlegm, wheezing, shortness of breath and 'chest illness') and on a history 'ever' of metal fume fever (MFF). A variable 'respiratory symptoms' was defined by the presence of any of the current respiratory symptoms listed above with the exception of MFF. In addition, we asked participants about their smoking practice (current, past, never), about any occupational welding exposure prior to their start of employment at the yard and into their current use of respiratory protective equipment. Analyses only of the questionnaire responses on respiratory symptoms are presented here.

\section{Examination of medical records}

The yard has an on-site primary care medical facility for most employees. Since 2000 the staff at the medical centre have, using a standardised system, recorded a date and diagnostic code for each consultation; through a unique employee number, this information can be linked to the payroll that includes information on job titles and dates of employment. The former was collapsed into five categories of exposure: welders, manual labourers (non-welding shipyard manual workers such as dock workers, mechanics, security staff, electricians, drivers etc.), painters/blasters, firemen and office workers. Based on their job titles, we further divided the welding group into 'high' (welders), 'medium' (platers and boilermakers) and 'low' (pipefitters) exposure categories.

From these sources we downloaded anonymised diagnostic and employment information for every consultation with male employees that took place between 1 January 2000 and 31 December $2011(n=259422)$ and was recorded under one of the following diagnostic codes: bronchitis, dyspnoea, influenza, pneumonia, sinusitis, sore throat and upper respiratory tract infections (URTIs). Consultations by the same individual that took place within 28 days of another with the same code were excluded, being considered as follow-up visits. In this way, we analysed 103840 'incident infections' in a cohort of 15954 employees working at any time in the 11 year period. The great majority (98\%) of these were coded as 'URTI'.

\section{Statistical analyses}

For the cross-sectional survey data, proportions of participants who reported current respiratory symptoms were compared using the $\chi^{2}$ test. Initial explorative analysis revealed that categorisation based on job title used for sampling was not always accurate, as $32 \%$ of workers classified as 'welders' did not report any current welding in the survey questionnaire. For this reason, we used questionnaire information on current welding to identify welders and other manual labourers for the main analysis and, with information on self-reported hours of welding, to identify three levels of exposure: high ( $>5 \mathrm{~h}$ daily), medium (3-5 h) and low (1-2 h). We used multivariable logistic regression to examine the relationship between respiratory symptoms and welding exposure after adjustment for a set of potential confounders identified prior to analysis (age, country of origin, smoking status and season of survey). Subsequently, we stratified the analysis by season, for which we pre-defined two categories: winter (October to March) and summer (April to September) based on climate data collected in Dubai, where the daily average minimum and maximum temperatures in January are $17^{\circ} \mathrm{C}$ and $25^{\circ} \mathrm{C}$, compared with $33^{\circ} \mathrm{C}$ and $42^{\circ} \mathrm{C}$ in July. ${ }^{6}$ We explored effect modification by season by adding an interaction term to the model.

The analyses for the historical medical records were restricted to men. We estimated incidence rate ratios (IRRs) for the number of consultations for each of the occupational exposure categories using a count regression model, with a negative binomial distribution due to overdispersion. We accounted for varying exposure time (tenure) by using the 'offset' function in STATA. The IRRs were adjusted for age, country of origin and season. Non-welding manual workers were used as a comparison group. In the same way as in the cross-sectional data analysis, we explored effect modification by season. We performed a sensitivity analysis by varying the interval to distinguish first consultations from follow-up visits. All analyses were conducted with STATA V.13 (StataCorp, 2013).

\section{Ethical approval}

This work was approved by the Imperial College Research and Ethical Committee (ICREC_12_3_1 and ICREC_12_3_2). Each participant in the cross-sectional survey provided written, informed consent. No participants' consent was required for the analysis of anonymised medical consultation data.

\section{FINDINGS}

\section{Cross-sectional survey}

Employees were surveyed over a 12-month period between January 2013 and December 2013; 213 (40\%) employees were surveyed in winter months. Although the prevalence of current respiratory symptoms was low overall (14\%, 76 of 529), those who were surveyed in winter months reported these far more frequently (figure 1), a pattern that was statistically significant ( $\mathrm{p}$ value for trend $<0.001$ ).

The comparison of welders with manual labourers is summarised in table 1 . The frequency matching ensured approximately equal distributions of age and country of origin. Of those classified as manual labourers, $21 \%$ had a welding 
Figure 1 Prevalence of reported respiratory symptoms by month of survey.

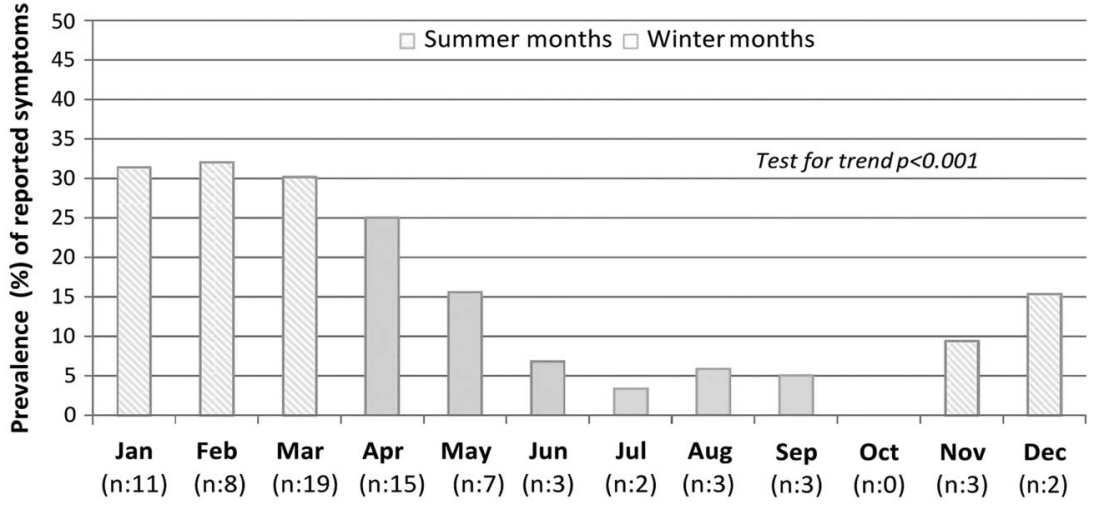

Month of recruitment occupation prior to their employment at the yard. The vast majority of welders $(219,80 \%)$ reported using manual metal arc welding. Overall, about one in four reported current smoking, but there were no differences in smoking habit between welders and manual labourers.

Overall, across the full year of survey, current respiratory symptoms were reported more frequently by welders than other manual labourers although statistical significance was borderline $(p=0.058)$. Welders who were surveyed in winter months, however, were significantly more likely to report respiratory symptoms $(26 \%)$ than manual labourers (14\%) surveyed at the same time of the year $(p=0.029)$. This difference was not apparent in participants who were surveyed in summer months.

Table 1 Comparison of respiratory symptoms and potentially confounding variables between welders and non-welding manual labourers who took part in the cross-sectional survey

\begin{tabular}{|c|c|c|c|c|}
\hline & $\begin{array}{l}\text { All } \\
\mathrm{N}=529\end{array}$ & $\begin{array}{l}\text { Welders } \\
\mathrm{n}=274\end{array}$ & $\begin{array}{l}\text { Manual labourers } \\
\mathrm{n}=\mathbf{2 5 5}\end{array}$ & p Value \\
\hline Age-median (IQR) & $38(33 ; 45)$ & $38(32 ; 44)$ & $40(33 ; 46)$ & 0.058 \\
\hline \multicolumn{5}{|l|}{ Nationality: n (\%) } \\
\hline Bangladesh & $262(50)$ & $146(53)$ & $116(45)$ & \\
\hline India & $182(34)$ & $86(31)$ & $96(38)$ & \\
\hline Philippines & $53(10)$ & $30(11)$ & $23(9)$ & \\
\hline Pakistan & $32(6)$ & $12(4)$ & $20(8)$ & 0.101 \\
\hline \multicolumn{5}{|l|}{ Smoking: n (\%) } \\
\hline Current & $129(24)$ & $67(24)$ & $62(24)$ & \\
\hline Past & $65(12)$ & $34(12)$ & $31(12)$ & \\
\hline Never & $334(63)$ & $173(63)$ & $161(63)$ & 0.997 \\
\hline Pack-years current smokers: median (IQR) & $4(2 ; 8)$ & $5(2 ; 8)$ & $4(2 ; 7)$ & 0.474 \\
\hline Tenure in years: median (IQR) & $5.9(4.5 ; 8)$ & $5.7(4.4 ; 7.1)$ & $6.2(4.5 ; 10.5)$ & 0.018 \\
\hline Past welding: $\mathrm{n}(\%)$ & $245(46)$ & $192(70)$ & $53(21)$ & $<0.001$ \\
\hline Years of past welding: median (IQR) & $5(6)$ & $5(6)$ & $4(4)$ & 0.121 \\
\hline Total years of welding: median (IQR) & $9(9)$ & $9(9)$ & - & - \\
\hline Daily hours* of arc welding: median (IQR) & $4(6)$ & $4(6)$ & - & - \\
\hline Welding exposure: $\mathrm{n}(\%)$ & & & - & - \\
\hline High $(>5 \mathrm{~h})$ & $121(44)$ & $121(44)$ & - & - \\
\hline Medium (3-5 h) & $43(16)$ & $43(16)$ & - & - \\
\hline Low $(<3 \mathrm{~h})$ & $110(40)$ & $110(40)$ & - & - \\
\hline Cutting and grinding: $\mathrm{n}(\%)$ & $302(57)$ & $230(84)$ & $72(28)$ & $<0.001$ \\
\hline Daily hours*: median (IQR) & $3(4)$ & $3(4)$ & $2(3)$ & 0.081 \\
\hline \multicolumn{5}{|l|}{ Breathing mask worn while welding $-n(\%)$} \\
\hline Always & $193(71)$ & $193(71)$ & - & \\
\hline Most of the time & $58(21)$ & $58(21)$ & - & \\
\hline Sometimes & $19(7)$ & $19(7)$ & - & \\
\hline Never & $3(1)$ & $3(1)$ & - & \\
\hline Symptoms—summer: n (\%) & $33(10)$ & $19(11)$ & $14(9)$ & 0.565 \\
\hline Symptoms—winter: $\mathrm{n}(\%)$ & $43(20)$ & $28(26)$ & $15(14)$ & 0.029 \\
\hline MFF一summer: $\mathrm{n}(\%)$ & $59(19)$ & $13(9)$ & $46(28)$ & $<0.001$ \\
\hline MFF-winter: n (\%) & $31(15)$ & $6(6)$ & $25(23)$ & $<0.001$ \\
\hline
\end{tabular}


Table 2 Demographic characteristics of male shipyard workers in the respiratory infection consultations cohort (2000-2011)

\begin{tabular}{|c|c|c|c|c|c|c|c|}
\hline & $\begin{array}{l}\text { Total } \\
\mathrm{N}=15954\end{array}$ & $\begin{array}{l}\text { Manual labourers } \\
\mathrm{n}=7207\end{array}$ & $\begin{array}{l}\text { Welders } \\
n=5865\end{array}$ & $\begin{array}{l}\text { Blaster/painter } \\
n=1496\end{array}$ & $\begin{array}{l}\text { Firemen } \\
n=363\end{array}$ & $\begin{array}{l}\text { Office workers } \\
n=1023\end{array}$ & p Value \\
\hline Age: median (IQR) & $32(27 ; 38)$ & $33(27 ; 39)$ & $32(28 ; 38)$ & $32(27 ; 37)$ & $30(26 ; 33)$ & $32(27 ; 39)$ & $<0.001$ \\
\hline Tenure in years-median (IQR) & $4(2 ; 8)$ & $4(2 ; 9)$ & $4(2 ; 7)$ & $4(2 ; 6)$ & $3(2 ; 5)$ & $4(2 ; 9)$ & $<0.001$ \\
\hline \multicolumn{8}{|l|}{ Nationality一n (\%) } \\
\hline Bangladesh & $2234(14)$ & $639(9)$ & $1285(22)$ & $150(10)$ & $137(38)$ & $23(2)$ & \\
\hline India & $6221(39)$ & $2891(40)$ & $2162(37)$ & $607(41)$ & $102(28)$ & $459(45)$ & \\
\hline Philippines & $4141(26)$ & $1646(23)$ & $1782(30)$ & $532(36)$ & $63(17)$ & $118(12)$ & \\
\hline Pakistan & $1451(9)$ & $895(12)$ & $310(5)$ & $144(10)$ & $8(2)$ & $94(9)$ & \\
\hline 50 others & 1907 (12) & $1136(16)$ & $326(6)$ & $63(4)$ & $53(15)$ & $329(32)$ & $<0.001$ \\
\hline Respiratory consultations: median (IQR) & $4(1 ; 9)$ & $3(1 ; 8)$ & $5(2 ; 11)$ & $4(2 ; 10)$ & $3(1 ; 6)$ & $2(1 ; 6)$ & $<0.001$ \\
\hline
\end{tabular}

MFF was reported more frequently by welders, with over a quarter ever experiencing it; the 19 non-welding workers (seven platers, 10 pipe fitters, one scaffolder and one welder foreman) who reported MFF were likely, because of their job title, to have worked in close proximity to welders. The difference between welders and manual labourers was not affected by season of survey.

Multiple regression analysis resulted in an OR of 2.22 (95\% CI 1.01 to 2.88 ) for the association between respiratory symptoms and welding. A subgroup analysis by season showed an increased OR for workers who were surveyed in winter $(2.58$; 1.20 to 5.58$)$ but not for those surveyed in summer $(1.66 ; 0.82$ to 3.38 ), although a formal test for interaction was not statistically significant $(p=0.117)$. Stratification by smoking status showed no difference between ever-smokers $(1.96 ; 0.76$ to $5.13)$ and never-smokers $(1.66 ; 0.82$ to 3.38$)$. There was no difference in the overall OR when the above analysis was performed using the welding exposure categories with ORs of 1.64 (0.86 to 3.13$), 2.61$ (1.10 to 6.16$)$ and 1.50 (0.77 to 2.91$)$ for high, medium and low level of exposure, respectively.

\section{Examination of medical records}

The median age of the cohort was 32 years (range: 19 to 71 ) with no substantial differences between the occupational groups (table 2). The median tenure of employment at the yard was 4 years, with only firemen having a slightly lower average of 3 years. There was significant variation in workers' country of origin in the different job groups with high proportions of welders from Bangladesh and other manual labourers from Pakistan. Employees from these countries also had higher than average numbers of consultations than those from other countries (median: 6 vs 2 , p<0.001). Only 15 workers had consultations for pneumonia and of these, almost half $(7,47 \%)$ were reported by other manual labourers and a third by welders (5, 33\%). The adjusted IRR for pneumonia was 1.36 (0.31 to 5.08), showing no statistically significant difference between welders and manual labourers.

There was a seasonal trend (figure 2) in consultations for respiratory infections with the highest rates in winter months. The adjusted IRRs indicated increased consultation rates for all groups of non-office workers (table 3), with the highest figures for welders and paint/blast workers and evidence for an 'exposure-response' pattern among welders with different levels of exposures to welding.

After stratification by season, the IRRs in welding employees were higher in winter than summer months; a test for interaction was statistically significant $(\mathrm{p}<0.001)$. The IRR among 'blast/paint' workers did not change by season. In sensitivity analyses, after extending the consultation cut-off to $35,42,49$ and 56 days, the pattern observed in the overall analysis remained unchanged. In a further analysis, consultations for URTIs were excluded; the resultant risk estimates revealed that welders still had a higher rate of consultations than manual labourers (overall IRR $1.57 ; 1.11$ to 2.21 ), an increase confined to consultations in the winter (IRR $1.57 ; 1.06$ to 2.32 ) and absent in summer (IRR $0.63 ; 0.29$ to 1.40 ).

\section{DISCUSSION}

The fieldwork for the cross-sectional survey spanned a period of 12 consecutive months and revealed a strong seasonal pattern in the reporting of current respiratory symptoms with substantially higher prevalences in winter months. Such symptoms were
Figure 2 Consultations for respiratory infections by month, 2000-2011.

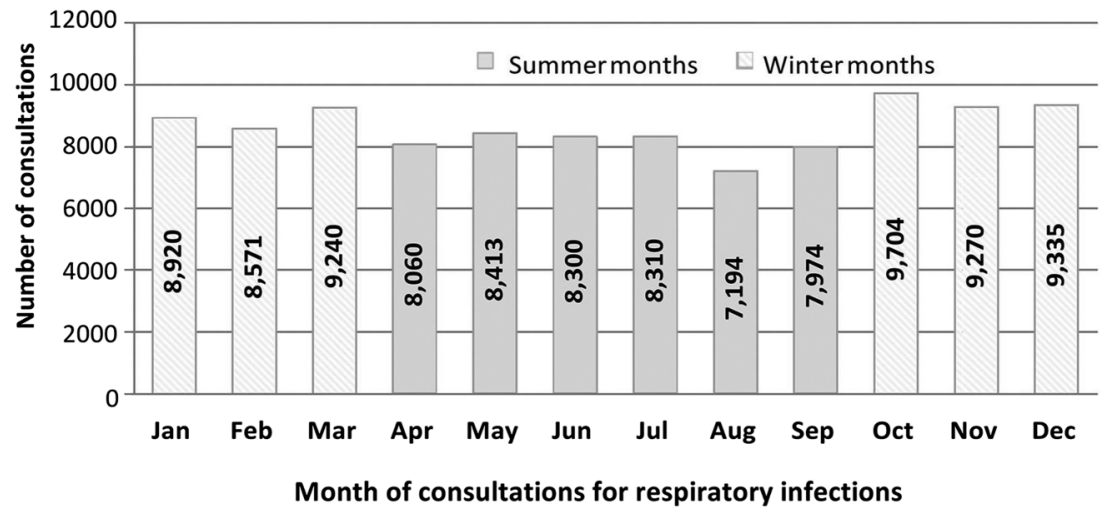

Marongiu A, et al. Thorax 2016;71:601-606. doi:10.1136/thoraxjnl-2015-207912 
Table 3 IRR for respiratory infection consultations (men only) by occupational group and season of consultation, 2000-2011

\begin{tabular}{|c|c|c|c|c|c|c|}
\hline \multirow[b]{2}{*}{ Occupation groups } & \multicolumn{2}{|l|}{ Overall } & \multicolumn{2}{|l|}{ Winter } & \multicolumn{2}{|l|}{ Summer } \\
\hline & Adjusted IRR* & $\mathrm{p}$ Value & Adjusted IRR† & $p$ Value & Adjusted IRR† & $\mathrm{p}$ Value \\
\hline Manual labourers & 1 & & 1 & & 1 & \\
\hline Welders (all) & $1.45(1.40 ; 1.50)$ & $<0.001$ & $1.47(1.42 ; 1.52)$ & $<0.001$ & $1.33(1.23 ; 1.44)$ & $<0.001$ \\
\hline High & $1.48(1.47 ; 1.61)$ & $<0.001$ & $1.58(1.50 ; 1.67)$ & $<0.001$ & $1.32(1.19 ; 1.47)$ & $<0.001$ \\
\hline Medium & $1.48(1.42 ; 1.55)$ & $<0.001$ & $1.49(1.43 ; 1.57)$ & $<0.001$ & $1.40(1.27 ; 1.55)$ & $<0.001$ \\
\hline Low & $1.22(1.15 ; 1.29)$ & $<0.001$ & $1.22(1.15 ; 1.30)$ & $<0.001$ & $1.24(1.09 ; 1.40)$ & $<0.001$ \\
\hline Blast/paint workers & $1.45(1.38 ; 1.53)$ & $<0.001$ & $1.46(1.37 ; 1.54)$ & $<0.001$ & $1.46(1.29 ; 1.64)$ & $<0.001$ \\
\hline Firemen & $1.01(0.91 ; 1.12)$ & 0.808 & $0.96(0.85 ; 1.08)$ & 0.587 & $1.24(1.02 ; 1.51)$ & 0.034 \\
\hline Office workers & $0.80(0.75 ; 0.85)$ & $<0.001$ & $0.79(0.73 ; 0.85)$ & $<0.001$ & $0.82(0.71 ; 0.95)$ & 0.008 \\
\hline
\end{tabular}

reported most frequently by those who were employed in the yard as welders, a difference that was apparent only for those surveyed between October and March and unexplained by differences in age, smoking or country of origin. These findings were supported by our analyses of medical consultations for probable respiratory infections that were most common among welders, again especially in winter months. Further analysis indicated that higher rates of consultations among welders were not solely explained by the occurrence of URTIs but by a broader range of diagnoses. We suggest that these observations are indirect evidence that there may be a heightened risk of respiratory infections among welders that is over and beyond their established risk of pneumococcal disease. Flu surveillance data from the United Arab Emirates show that seasonal flu peaks, as in most countries in the northern hemisphere, in the winter months from January till March. ${ }^{7}$

Few previous studies of welders have reported findings that can readily be compared with ours. In a survey of Danish shipyard workers, ${ }^{8}$ comprising 2660 welders and 881 electricians, welders were more likely $(16 \%)$ than manual labourers $(8 \%$, difference $\mathrm{p}<0.001)$ to report 'colds at least once a month'; the prevalence in 'high-exposed' welders was greater (17\%) than in those with 'low' exposure (13\%). Similarly, in a smaller survey of Finnish welders working in engineering workshops ${ }^{9}$ frequent colds were reported by a higher proportion (23\%) than in a non-welding referent group (11\%), a difference that was also statistically significant. Welders in a New York plant manufacturing construction equipment ${ }^{10}$ were more likely than their non-welding co-workers to report that 'colds go to chest'; this difference however was confined to non-smokers and was not statistically significant. Finally, a small study looking at work absences in a petrochemical plant found that welders compared with controls had a greater inception rate and longer annual duration of absence spells attributable to lower respiratory tract diseases. ${ }^{11}$ None of these reports included findings stratified by season. Numerous other surveys of welders have reported on non-specific respiratory symptoms such as 'bronchitis', 'catarrh' or 'breathlessness', but it is impossible to know to what extent these reflect infective episodes.

The mechanisms whereby welding fume might increase susceptibility to respiratory infection are probably several. Free iron serves as a nutrient for microorganisms, ${ }^{12}{ }^{13}$ can facilitate penetration of a pathogen deeper into the lung than it could normally achieve ('carrier' effect) and activate a pneumococcal iron-dependent transcriptional regulator which promotes the expression of genes required for bacterial survival and virulence in the host. ${ }^{14}$ In addition, iron can modify cytokine signalling and the activation of recruited inflammatory cells ${ }^{15} 16$ and promote apoptosis via iron-catalysed oxidative stress (via generation of reactive hydroxyl radicals), impairing the activation of neutrophils and normal immune responses such as the migration of macrophages in the airways. ${ }^{17}$ Iron can also induce oxidative-mediated epithelial damage, or activate alveolar macrophages to establish a systemic eosinophilia and basophilia through cytokine signalling. ${ }^{19}$ Alternatively, the ultrafine particles-of whatever composition-produced in the welding process may increase a pathogen's virulence and decrease a host's defence, in particular by directly impairing respiratory epithelium mucociliary function, bacterial phagocytosis by macrophages and native immune cell responses. ${ }^{20}$ Furthermore, animal studies have shown how inhalation of non-metals such as fluoride (present in welding electrode fluxes) suppresses lung antibacterial mechanisms in rats. ${ }^{21}$

We recognise a number of important limitations to this report. We did not set out to study infections but, rather, a broad range of current respiratory symptoms and lung function in a population of shipyard welders and other employees. To do this we used a simple, self-completed questionnaire, translated into a number of languages, that was designed to enquire, in the present tense, into the existence of a non-specific range of symptoms such as cough, wheeze, shortness of breath and chest 'illness'. The strong seasonal pattern in reporting suggests that positive responses were indicative of infective symptoms but we have no direct evidence that that is the case. Our analysis of medical consultations was reliant on diagnostic coding which is inevitably subjective and open to the potential for information bias, and on consultation behaviour which may vary systematically between different employment groups and between different nationalities, although our findings in relation to welders were independent of country of origin. One further limitation of the historical data was the absence of any information on smoking habits. Our finding of an increased risk of pneumonia in welders-as reported by others-was not statistically significant but this was not the primary outcome of interest and the study was not powered appropriately.

Finally, in all analyses, we used job title as a measure of 'exposure' and while this is almost certainly accurate for the great majority of employees there may have been some misclassification among those employed in non-office roles. In this respect, it is interesting to note the high rates of consultations 
for respiratory infections among 'blast/paint' workers. These workers are responsible for preparing ship hulls and other parts for repainting, using high pressure water and grit to remove large areas of corrosion and dirt from steel surfaces with probably high exposures to metal (iron) dusts; steel is one of the several grit materials used, the remainder being copper slag and garnet. Notably, although we observed an increased risk in this group it was not affected by season of consultation, suggesting perhaps only an irritant rather than infectious aetiology.

It seems clear from evidence collected elsewhere that welders have, through their work, a heightened susceptibility to pneumococcal infection but there is little evidence that this extends to other respiratory infections. Our findings suggesting that respiratory symptoms and consultations for respiratory infection in welders are more common in winter may indicate an enhanced vulnerability to a broad range of infections. Further research should be undertaken to include direct, seasonal microbiological surveillance in welders in relation to their welding fume exposure. If these results are confirmed, this would have important implications for the occupational healthcare of a very large, global workforce.

Acknowledgements We acknowledge and are very grateful for the hard work of DryDocks World medical centre and for the cooperation shown by the yard's management and owners.

Contributors $\mathrm{AM}, \mathrm{PC}$ and $\mathrm{OH}$ contributed to the study design. $\mathrm{AM}, \mathrm{OH}, \mathrm{AA}, \mathrm{SB}$, $B G$ and NI participated in the implementation of the cross-sectional study. $A M$ and AA double entered the cross-sectional questionnaire data. AA collated the historical medical data and AM performed the data linkage, designed and conducted all statistical analyses. CM and SS provided statistical assistance. AM wrote the first draft of the manuscript; all other authors critically reviewed it and approved the final version.

Competing interests None declared.

Ethics approval Imperial College Research Ethics Committee.

Provenance and peer review Not commissioned; externally peer reviewed.

\section{REFERENCES}

1 Coggon $\mathrm{D}$, Inskip $\mathrm{H}$, Winter $\mathrm{P}$, et al. Lobar pneumonia: an occupational disease in welders. Lancet 1994;344:41-3.
2 Wong A, Marrie TJ, Garg S, et al. Welders are at increased risk for invasive pneumococcal disease. Int I Infect Dis 2010;14:e796-9.

3 Wergeland E, Iversen BG. Deaths from pneumonia after welding. Scand I Work Environ Health 2001;27:353.

4 Torén K, Qvarfordt I, Bergdahl IA, et al. Increased mortality from infectious pneumonia after occupational exposure to inorganic dust, metal fumes and chemicals. Thorax 2011;66:992-6.

5 Palmer KT, Poole J, Ayres JG, et al. Exposure to metal fume and infectious pneumonia. Am J Epidemiol 2003;157:227-33.

6 National Bureau Of Statistics. Climate 2013, in Climate Statistics. UAE: National Bureau Of Statistics, 2014.

7 Health Authority-Abu Dhabi. Communicable Diseases Bulletin: Quarterly Summary Report; First Quarter - 2013 (Jan - Mar). UAE: Health Authority-Abu Dhabi, 2013;4:1-24. http://www.haad.ae/haad/tabid/1177/Default.aspx

8 Groth M, Lyngenbo O. Respiratory symptoms in Danish welders. Scand I Soc Med 1989;17:271-6.

9 Anttipoika M, Hassi J, Pyy L. Respiratory-diseases in arc welders. Int Arch Occup Environ Health 1977;40:225-30.

10 Keimig DG, Pomrehn PR, Burmeister LF. Respiratory symptoms and pulmonary function in welders of mild steel: a cross-sectional study. Am J Ind Med 1983:4:489-99.

11 Fawer RF, Gardner AW, Oakes D. Absences attributed to respiratory diseases in welders. Br J Ind Med 1982;39:149-52.

12 Otto BR, Verweij-Van Vught AM, Maclaren DM. Transferrins and heme-compounds as iron sources for pathogenic bacteria. Crit Rev Microbiol 1992;18:217-33.

13 Tai SS, Lee CJ, Winter RE. Hemin utilization is related to virulence of streptococcus-pneumoniae. Infect Immun 1993;61:5401-5.

14 Gupta R, Bhatty M, Swiatlo E, et al. Role of an iron-dependent transcriptional regulator in the pathogenesis and host response to infection with Streptococcus pneumoniae. PLOS ONE 2013;8:e55157.

15 Antonini JM, Stone S, Roberts JR, et al. Effect of short-term stainless steel welding fume inhalation exposure on lung inflammation, injury, and defense responses in rats. Toxicol Appl Pharmacol 2007;223:234-45.

16 Blanc PD, Boushey HA, Wong H, et al. Cytokines in metal fume fever. Am Rev Respir Dis 1993;147:134-8.

17 Erdely A, Antonini JM, Young S-H, et al. Oxidative stress and reduced responsiveness of challenged circulating leukocytes following pulmonary instillation of metal-rich particulate matter in rats. Part Fibre Toxicol 2014;11:34.

18 Zeidler-Erdely PC, Erdely A, Antonini JM. Immunotoxicology of arc welding fume: worker and experimental animal studies. J Immunotoxicol 2012;9:411-25.

19 Antonini JM, Leonard SS, Roberts JR, et al. Effect of stainless steel manual metal arc welding fume on free radical production, DNA damage, and apoptosis induction. Mol Cell Biochem 2005;279:17-23.

20 Ghio AJ. Particle exposures and infections. Infection 2014;42:459-67.

21 Yamamoto $S$, Katagiri $\mathrm{K}$, Ando $\mathrm{M}$, et al. Suppression of pulmonary antibacterial defenses mechanisms and lung damage in mice exposed to fluoride aerosol. J Toxicol Environ Health A 2001;62:485-94. 